NASA Technical Memorandum 100571

\title{
ROBUST ADAPTIVE REGULATION WITHOUT PERSISTENT EXCITATION
}

Rogelio Lozano-Leal

May 1988

\section{LBGAGiV COPY}

\author{
JUL 11988 \\ LANGLEY RESEAPCH CENTER \\ - tumpton, VIRGinia
}

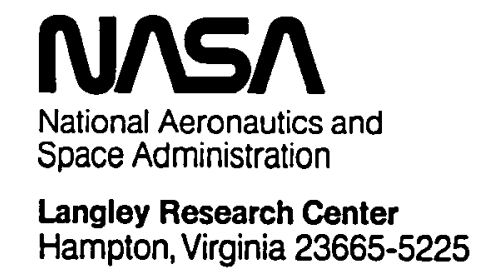




\section{Summary}

This paper presents a globally convergent adaptive regulator for minimum or nonminimum phase systems subject to bounded disturbances and unmodeled dynamics. The control strategy is designed for a particular input-output representation obtained from the state space representation of the system. The leading coefficient of the new representation is the product of the observability and controllability matrices of the system. The controller scheme uses a Least Squares identification algorithm with a dead zone. The dead zone is chosen to obtain convergence properties on the estimates and on the 'covariance matrix' as well. This allows the definition of modified estimates which secure well-conditioned matrices in the adaptive control law. Explicit bounds on the plant output are given.

\section{Introduction}

Adaptive control techniques have been extensively studied for over a decade. The development of the theory has led to a better understanding and improvement of the performance of adaptive algorithms. Nevertheless, owing to the difficulties encountered when dealing with nonminimum phase systems, most of the treatment is confined to minimum phase systems.

Discrete time minimum phase systems have the appealing property that boundedness of the output implies boundedness of the input. This property has been thoroughly exploited in the convergence analysis of adaptive schemes in which attention may be exclusively focused to obtain bounds on the plant output. A fortunate coincidence is that prediction error identification methods like Least Squares and projection algorithms are also focused on the plant output in the sense that they provide plant model estimates that adequately predict the plant output. These two facts allow one to compute a control sequence that produces an arbitrary desired output regardless of the controllability of the plant model estimate (refs. 1 through 10).

Discrete time systems having nonminimum phase characteristics may appear in many different ways. For instance, all continuous systems having a relative degree greater than two give rise to nonminimum phase models when sampled at a fast rate with zero order hold input (ref. 11). In that paper it was also found that the pulse transfer function corresponding to $1 / \mathrm{s}^{\mathrm{n}}$ has zeros on or outside the unit circle for $n \geq 2$. This indicates the restricted scope of application of those control laws for discrete time systems which involve cancellation of discrete zeros. It also underlines the importance of the study of adaptive control for nonminimum phase plants. 
Parameter estimation methods like Least Squares and projection algorithms are derived from an optimization problem formulation considering basically the output prediction error. It is not surprising that the plant model estimate obtained in such a way may not retain some important characteristics of the process like controllability, stability, delay, etc. .. Lack of controllability has been a stumbling block for the development of adaptive control of nonminimum phase systems. Unlike the minimum phase case, adaptive control for nonminimum phase plants require controllability of the plant estimate in order to assure that both plant output and input remain bounded.

This problem has been addressed from two different perspectives: one relying on persistent excitation ( P.E. ) and the other not requiring P.E.. In the former an external signal having a minimum amplitude and a minimum number of spectral lines is introduced in the control loop. Such a signal will assure that a certain P.E. condition on the plant input and output will be satisfied. In the deterministic case, $1 . e$. when there is no noise in the plant, P.E. on the plant signals will in turn assure convergence of the estimates to the true parameter values which will guarantee boundedness of all the signals in the control loop (refs. 12 through 15). This approach not only secures boundedness of all the signals but also exponential stability.

However, in the presence of bounded disturbances or unmodeled dynamics the amplitude and frequency richness of the external signal should be large enough to secure P.E. of the plant input and output and prevent the noise in the closed loop from counteracting the external signal effect. Introducing an external signal of such a size into the plant may conflict with the control objective of having a small output tracking error. On the other hand, from the practical point of view, it is not always feasible or desirable to introduce extra noise into the system. For these reasons adaptive control not relying on persistency of excitation is an important alternative approach. Furthermore, adaptive stabilization without resorting to any $P . E$. condition will pave the way to a better understanding of the use of P.E. in the control loop. Indeed, if desired, a probing signal with a tolerable amount of P.E. could be introduced in the system to improve a given performance index, but not to guarantee stability.

In the design of adaptive controllers whose stability does not rely on P.E. one is readily confronted with the problem of obtaining a controllable plant estimate. As pointed out above, minimization of performance indices on the output prediction error may not suit our control objectives. However, Least Squares type identification algorithms offer some freedom to modify the parameter estimates. As is well known, the "covariance matrix" acts as a memory and can be used to measure the parameter estimation error. It can also be used to define a modified 
parameter estimate that will essentially preserve the convergence properties of the original parameter estimate. Such a modified estimate is obtained by adding any linear combination of columns of the " covariance matrix " to the current estimate. The weights on the linear combination are arbitrary and can be chosen to our advantage. This technique was used in the design of adaptive control schemes in the deterministic case in references 16 and 17 wherein different modifications were proposed to the standard Least Squares identification algorithm. These modifications secure a nonsingular Sylvester resultant matrix obtained using the modified parameter estimates. The Sylvester resultant matrix appears in pole placement techniques and is nonsingular if and only if the associated system is controllable (ref. 18). Other modifications have been studied in reference 19 but the proposed algorithms are less explicit than those in references 16 and 17. Another interesting approach is presented in reference 19 where the problem is solved for the class of plants whose parameters belong to a convex region in the parameter space where the plant is pointwise stabilizable. Further studies are required to completely assess and characterize the plants belonging to that class.

The modifications proposed to date in the literature have not been proved to secure a uniform, strictly positive lower bound for the singular values of the Sylvester matrix obtained using the modified estimates. Since the control input depends on the inverse of the Sylvester matrix, the size of that lower bound plays an important role in the assessment of the robustness capabilities of the adaptive control scheme. Clearly this problem is shared by any control law making use of the certainty equivalence principle.

This paper presents a robust adaptive regulator for discrete time systems in the presence of bounded disturbances and unmodeled dynamics. The system is not restricted to be minimum phase and the class of unmodeled dynamics considered is the same as in reference 8 . The control objective is to achieve plant output regulation.

The control strategy has been devised for a particular input-output representation of the system. This representation is obtained from the state space representation of the plant and has the key feature that the matrix coefficient multiplying the sequence of inputs is the product of the system observability and controllability matrices. That product shall be refered to in the paper, as the leading coefficient. Therefore the leading coefficient can be directly identified and thus, the effect of any modification of the estimates on the controllability of the plant model estimate will appear more clearly. A Least Squares identification algorithm with a particular dead zone is used. The width of the dead zone is such that convergence properties can be deduced, not only on the output prediction error but on the " 
covariance matrix " as well. This leads to an explicit definition of a modified parameter estimate for which the singular values of the leading coefficient have a. uniform, strictly positive lower bound. Explicit bounds for the plant output in the limit are also presented.

The material that follows presents a discussion of a particular input-output representation of the system and a simple output feedback control law that achieves state regulation around the origin; the proposed control scheme and its obtained convergence properties.

\section{Symbols}

$\begin{array}{ll}E & \text { prediction error vector } \\ F & \text { covariance matrix } \\ L & \text { a matrix such that } F=L^{T} \\ n & \text { order of the plant } \\ u(t) & \text { plant input } \\ x(t) & \text { state vector } \\ y(t) & \text { plant output } \\ W_{k} & \text { augmented error } \\ \delta_{k} & \text { normalized disturbance bound } \\ \tilde{G} & \text { controllability matrix } \\ 0 & \text { observability matrix } \\ \theta & \text { parameters vector } \\ \theta & \text { modified parameters estimate } \\ \tilde{\theta} & \text { parametric distance } \\ \theta_{0} & \text { initial parameters estimate } \\ \phi & \text { measurement vector } \\ \lambda_{k} & \text { forgetting factor }\end{array}$

State Regulation via Output Feedback

Consider the following state space representation of a single-input single-output discrete-time system 


$$
\begin{aligned}
& x(t+1)=A x(t)+b u(t)+b^{\prime} v^{\prime}(t) \\
& y(t)=c^{T} x(t)+v^{\prime \prime}(t)
\end{aligned}
$$

where $x$ is the $(n \times 1)$ state vector, $u$ and $y$ are the plant input and output, $A, b, b^{\prime}$ and $c$ are matrices and vectors of appropiate dimensions, and, $v^{\prime}$ and $v^{\prime \prime}$ are disturbances.

The control strategy proposed in this paper is based on the input-output representation presented in the following Lemma.

Lemma 1- The plant output and input in equation (1) satisfy the following input-output representation

$$
Y(t+2 n)=G U(t+n)+B U(t)+D Y(t)+B^{\prime} U(t-n)+N(t+2 n)
$$

where

$$
\begin{aligned}
& U^{T}(t)=[u(t), \ldots, u(t+n-1)] \\
& Y^{T}(t+n)=[y(t), \ldots, y(t+n-1)] \\
& B=06
\end{aligned}
$$

with

$$
\begin{aligned}
& O=\left[\begin{array}{l}
c^{T} \\
c^{T} A \\
c^{T} A^{n-1}
\end{array}\right] ; \quad C=\left[A^{n-1} b, \ldots A b, b\right] \\
& D=O A^{2 n} O^{-1} \\
& B^{\prime}=O A^{n} \mathscr{C}-O A^{2 n} O^{-1} G \\
& G=\left[\begin{array}{lll}
O{ }^{T} b & \\
c^{T} b & \\
c^{T} A^{n-2} b & \ldots c^{T} b & 0
\end{array}\right]
\end{aligned}
$$




$$
\begin{aligned}
& N(t+2 n)=O Q^{\prime} V^{\prime}(t)+G^{\prime} V^{\prime}(t+n)+V^{\prime \prime}(t+n)- \\
& -O A^{2 n} O^{-1}\left[G^{\prime} V^{\prime}(t-n)+V^{\prime \prime}(t-n)\right]+O A^{n^{\prime}} C^{\prime} V^{\prime}(t-n)
\end{aligned}
$$

$6^{\prime}$ and $G^{\prime}$ are obtained by replacing $b$ by $b^{\prime}$ in the expressions for $\mathscr{C}$ and $G$ respectively. $V^{\prime}$ and $V^{\prime \prime}$ are defined the same way as $U$ in equation (3.a).

The proof is given in Appendix A.

The input-output relationship in equation 2 is an alternative representation of the system (eqn. 1) that has some particular characteristics. If the system in equation 1 is reachable and observable then $B$ in equation 3.c is nonsingular. On the other hand $G$ in equation $3 . \mathrm{g}$ is always singular and for this reason we reserved the name leading coefficient for $B$ and not for $G$. It can also be noted that $Y(t+n)$ does not appear in equation 2 and this will allow definition of a very simple control law.

Let $U_{k}$ denote $U$ at time $t=2 k n, k=0,1,2, \ldots$. Assume

$$
U(t-n)=0 \text { for } t=2 k n
$$

Note that $U(t+n)$ is also equal to zero for $t=2 k n$ because $U(2 k n+n)=U(2(k+1) n-n)$. Then equation 2 can be written as

$$
\mathrm{Y}_{\mathrm{k}+1}=\mathrm{DY}_{\mathrm{k}}+\mathrm{BU}_{\mathrm{k}}+\mathrm{N}_{\mathrm{k}+1}
$$

Consider the control strategy defined by equation 4 and the following

$$
\mathrm{U}_{\mathrm{k}}=-\mathrm{B}^{-1} \mathrm{DY} \mathrm{k}_{\mathrm{k}}
$$

Note that $Y_{k}$ depends on output measurements up to time $t=2 k n-1$ as can be seen from equation 3.b. Then the control input in equations 4 and 6 leads to 


$$
\mathrm{Y}_{\mathrm{k}+1}=\mathrm{N}_{\mathrm{k}+1}
$$

Since $Y(t+2 n)=N(t+2 n)$ and $U(t+n)=0$ for $t=2 k n, x(t+n)$ is exclusively bounded by the disturbances $N(t+2 n), V^{\prime}(t+n)$ and $V^{\prime \prime}(t+n)$ for time $t=2 k n$ (eqn. A.3). Since $U(t+n)=0, x(t+2 n)$ will be bounded by $x(t+n)$ and $V^{\prime}(t+n)$ for $t=2 k n$ ( eqn. A.2).

The proposed control strategy can also be interpreted as an alternated use of an n-step dead beat observer and an n-step dead beat controller. It is prefered not to increase the complexity of the control scheme at this stage to simplify the convergence analysis of the corresponding adaptive scheme which follows.

\section{Adaptive regulation}

We will assume that equation 4 holds so that the system is represented by equation 5 which can also be rewritten as

$$
\mathrm{Y}_{\mathrm{k}+1}=\theta \phi_{\mathrm{k}}+\mathrm{N}_{\mathrm{k}+1}
$$

where

$$
\begin{aligned}
& \theta=[B: D] \\
& \phi_{k}^{T}=\left[U_{k}^{T}: Y_{k}^{T}\right]
\end{aligned}
$$

Consider the class of disturbances satisfying

$$
\max _{n \leq \tau \leq 2 n} \quad\|N(2 k n+\tau)\| \leq \eta+\mu\left\|\phi_{k}\right\|
$$

The following a-priori knowledge on the plant is required

Assumption $1 \mathrm{n}$ and the upper bounds $\eta$ and $\mu$ in equation 9 are known Assumption 2 A lower bound $b_{0}$ is known such that 


$$
b_{0} I \leq B^{T} B
$$

Assumption 3 Matrices $\mathrm{F}_{0}>0$ and $\mathrm{H}_{0}>0$ defining an ellipsoid in the parameter space are known such that

$$
\left(\theta-\theta_{0}\right) F_{0}^{-1}\left(\theta-\theta_{0}\right)^{T} \leq H_{0}
$$

where $\theta_{0}$ is any initial parameter estimate. The quantities $b_{0}$ and $\mathrm{H}_{0}$ above are not actually required in the computation of the control law but the stability and the performance of the algorithm will depend on them.

The proposed adaptive scheme is now presented. The equations are given in the order they appear in the computation of the control input.

Description of the adaptive_control algorithm

Normalized variables

$$
\begin{aligned}
& x_{k-1}=\phi_{k-1} /\left(1+\left\|\phi_{k-1}\right\|\right) \\
& \underline{Y}_{k}=Y_{k} /\left(1+\left\|\phi_{k-1}\right\|\right)
\end{aligned}
$$

Prediction error

$$
E_{k}=\underline{Y}_{k}-\theta_{k-1} x_{k-1}
$$

Least Squares with dead zone 


$$
\begin{aligned}
& w_{k}^{2}=E_{k}^{T} E_{k}+x_{k-1}^{T} F_{k-1}^{2} x_{k-1} \\
& \delta_{k}=\mu+\eta /\left(1+\left\|\phi_{k-1}\right\|\right) \\
& \lambda_{k}=\left\{\begin{array}{cl}
0 & \text { if } w_{k}^{2} \leq \delta_{k}^{2}(1+\alpha) n \\
\frac{\alpha\left[\left|w_{k}\right|-\delta_{k} \frac{\left.((1+\alpha) n)^{1 / 2}\right]}{\left(1+x_{k-1}^{T}\right.} F_{k-1} x_{k-1}\right)\left|w_{k}\right|}{\text { otherwise }} &
\end{array}\right. \\
& \alpha>0 \\
& F_{k}=F_{k-1}-\frac{\lambda_{k} F_{k-1} x_{k-1} x_{k-1}^{T} F_{k-1}}{1+\lambda_{k} x_{k-1}^{T} F_{k-1} x_{k-1}} \\
& \theta_{k}=\theta_{k-1}+\lambda_{k} E_{k} x_{k-1}^{T} F_{k} \\
& \theta_{k}=\left[B_{k}(n \times n), D_{k}(n \times n)\right]
\end{aligned}
$$

Factors of $F_{k}$ and $B_{k}$

$$
F_{k}=L_{k} L_{k}^{T} \geq 0
$$

$$
B_{k}=Q_{k} S_{k} \quad ; \quad Q_{k}^{T} Q_{k}=I \quad \text { and } S_{k} \geq 0
$$

Modified parameter estimate 


$$
\begin{aligned}
& L_{k}=\left[\begin{array}{c}
L_{k}^{1}(n \times 2 n) \\
-\frac{k}{2}(n \times 2 n)
\end{array}\right] \\
& \beta_{k}=Q_{k} L_{k}^{1} \\
& \theta_{k}=\theta_{k}+\beta_{k} L_{k}^{T} \\
& \theta_{k}=\left[\underline{B}_{k}(n \times n), \underline{D}_{k}(n \times n)\right]
\end{aligned}
$$

Control input

$$
u_{k}=-\underline{B}_{k}^{-1} \underline{D}_{k} Y_{k}
$$

The Cholesky factorization in equation 18 and the polar decomposition in equation 19 can be carried out for any $F_{k} \geq 0$ and any $B_{k}$ respectively. The reader is refered to Appendix $B$ for a brief explanation on equation 19 and to References 29, 30 and 31 for a more detail presentation. In fact the parameter estimate modification in equation 22 is only required when $B_{k}$ in equation 17 is ill-conditionned, but for sake of simplicity of notation and since the modification will ultimately be required for convergence analysis, it is decided to leave it on all the time.

\section{Convergence Analysis}

The convergence analysis of the adaptive control scheme previously discussed is now presented. The main convergence properties of the control scheme are given in the following theorem. 
Theorem 1. Consider the system equation 2 whose disturbances belong to the class equation 9. Then subject to assumptions 1-3, the control law in equations 4 and 10 through 24 has the following 10 properties.

1) The plant parameter vector $\theta$ lies inside an ellipsoid centered at the parameter estimate as follows

$$
\tilde{\theta}_{k} F_{k}^{-1} \tilde{\theta}_{k}^{T} \leq H_{k}
$$

where $\tilde{\theta}$ is the parametric distance

$$
\tilde{\theta}_{\mathrm{k}}=\theta-\theta_{\mathrm{k}}
$$

and $H_{k}$ is given by the following expression (ref 32)

$$
H_{k}=H_{k-1}+\lambda_{k} \delta_{k}^{2} I-\frac{\lambda_{k} E_{k} E_{k}^{T}}{1+\lambda_{k} x_{k-1}^{T} F_{k-1} x_{k-1}}
$$

2) The forgetting factor $\lambda_{k}$ in equation 14 satisfies the following inequalities

$$
0 \leq \lambda_{k} \leq \alpha, \quad \lambda_{k} x_{k-1}^{T} F_{k-1} x_{k-1} \leq \alpha
$$

3) There exists a positive definite function $v_{k}$ satisfying

$$
\mathrm{v}_{\mathrm{k}} \leq \mathrm{v}_{\mathrm{k}-1}
$$

that is related to the size of the ellipsoid in equation 25 as follows

$$
V_{k}=\operatorname{tr}\left(F_{k}+H_{k}\right)
$$


4) The augmented error $w_{k}$ in equation 12 is bounded by $\delta_{k}$ in equation 13 as follows

$$
\lim _{k \rightarrow \infty} \sup \left(w_{k}^{2}-\delta_{k}^{2}(1+\alpha) n\right) \leq 0
$$

5) The covariance matrix $F_{k}$ in equation 15 converges.

6) $\lambda_{k}$ in equation 14 is such that

$$
\sum_{k=1}^{\infty} \lambda_{k} \delta_{k}^{2} \quad \text { is bounded }
$$

7) The parameter estimate vector $\theta_{k}$ converges and is bounded by

$$
\theta_{k} \theta_{k}^{T} \leq V_{0}^{2} I
$$

where $V_{0}$ is given in equation 30 for $k=0$. The matrices involved in the control law equation 24 satisfy

8)

$$
\underline{B}_{k}^{T} \underline{B}_{k} \geq \frac{b_{o}^{2} I}{4 v_{0}^{2}}
$$

9)

$$
\left(\underline{B}_{k}^{-1} \underline{D}_{k}\right)^{T}\left(\underline{B}_{k}^{-1} \underline{D}_{k}\right) \leq m I=\frac{\Delta}{=} \frac{16 v_{0}^{4} I}{b_{0}^{2}}
$$

10) If $\mu$ in equation 9 is small enough that $\exists \varepsilon>0$ such that

$$
\mu=\frac{1-\varepsilon}{[2 \mathrm{n}(1+\alpha)(1+m)]^{1 / 2}}>0
$$


with $\alpha$ and $m$ as in equations 14 and $34 \mathrm{a}$ respectively, then all the variables remain bounded and the plant output is bounded as follows

$$
\lim _{k \rightarrow \infty} \sup \left\{\left\|Y_{k}\right\|-[2 n(1+\alpha)]^{1 / 2}(\eta+\mu) / \varepsilon\right\} \leq 0
$$

Each of the 10 properties previously described will now be proved

Proof :

1) From equation 8 it is seen that

$$
\underline{Y}_{k}=\theta x_{k-1}+\underline{N}_{k}
$$

where $\underline{Y}_{k}$ and $x_{k-1}$ are given in equations $10 a$ and $10 \mathrm{~b}$ and

$$
\underline{N}_{k}=N_{k} /\left(1+\left\|\phi_{k-1}\right\|\right)
$$

In view of equations 9 and $13, \underline{N}_{k}$ satisfies

$$
\underline{N}_{k}^{T} \underline{N}_{k} \leq \delta_{k}^{2}
$$

From equations 16 and 26 it follows

$$
\tilde{\theta}_{k}=\tilde{\theta}_{k-1}-\lambda_{k} E_{k} x_{k-1}^{T} F_{k}
$$

From equation 15 and the matrix inversion Lemma (ref. 18)

$$
F_{k}^{-1}=F_{k-1}^{-1}+\lambda_{k} x_{k-1} x_{k-1}^{T}
$$

Combining equations 40 and 41 and noting that $F_{k}=F_{k}^{T} \geq 0$ 


$$
\begin{aligned}
\tilde{\theta}_{k} F_{k}^{-1} \tilde{\theta}_{k}^{T}= & \left(\tilde{\theta}_{k-1} F_{k}^{-1}-\lambda_{k} E_{k} x_{k-1}^{T} F_{k} F_{k}^{-1}\right)\left(\tilde{\theta}_{k-1}-\lambda_{k} E_{k} x_{k-1}^{T} F_{k}\right)^{T} \\
= & \tilde{\theta}_{k-1}\left(F_{k-1}^{-1}+\lambda_{k} x_{k-1} x_{k-1}^{T}\right) \tilde{\theta}_{k-1}^{T}-\lambda_{k} \tilde{\theta}_{k-1} x_{k-1} E_{k}^{T}+ \\
& -\lambda_{k} E_{k} x_{k-1}^{T} \tilde{\theta}_{k-1}^{T}+\lambda_{k}^{2} E_{k} x_{k-1}^{T} F_{k} x_{k-1} E_{k}^{T} \\
= & \tilde{\theta}_{k-1} F_{k-1}^{-1} \tilde{\theta}_{k-1}^{T}+\lambda_{k}\left(\tilde{\theta}_{k-1} x_{k-1}-E_{k}\right)\left(\tilde{\theta}_{k-1} x_{k-1}-E_{k}\right)^{T}+ \\
& +\lambda_{k} E_{k} E_{k}^{T}\left(\lambda_{k} x_{k-1}^{T} F_{k} x_{k-1}-1\right)
\end{aligned}
$$

From equations 11,26 and 37 it follows

$$
\tilde{\theta}_{k-1} x_{k-1}-E_{k}=\left(\theta-\theta_{k-1}\right) x_{k-1}-\left(\underline{Y}_{k}-\theta_{k-1} x_{k-1}\right)=-\underline{N}_{k}
$$

The quadratic term $x_{k-1}^{T} F_{k} x_{k-1}$ can be obtained from equation 15 as follows

$$
\begin{aligned}
x_{k-1}^{T} F_{k} x_{k-1} & =x_{k-1}^{T} F_{k-1} x_{k-1}-\frac{\lambda_{k}\left(x_{k-1}^{T} F_{k-1} x_{k-1}\right)^{2}}{1+\lambda_{k} x_{k-1}^{T} F_{k-1} x_{k-1}} \\
& =\frac{x_{k-1}^{T} F_{k} x_{k-1}}{1+\lambda_{k} x_{k-1}^{T} F_{k} x_{k-1}}
\end{aligned}
$$

or

$$
\lambda_{k} x_{k-1}^{T} F_{k-1} x_{k-1}-1=\frac{-1}{1+\lambda_{k} x_{k-1}^{T} F_{k-1} x_{k-1}}
$$

Introducing equations 43 and 44 into equation 42 and noting that (see eqn. 39)

$$
\underline{N}_{k} \underline{N}_{k}^{T} \leq \underline{N}_{k}^{T} \underline{N}_{k} I \leq \delta_{k}^{2} I
$$


gives

$$
\begin{aligned}
\tilde{\theta}_{k} F_{k}^{-1} \tilde{\theta}_{k}^{T}-\tilde{\theta}_{k-1} F_{k-1}^{-1} \tilde{\theta}_{k-1}^{T} & =\lambda_{k} \underline{N}_{k} \underline{N}_{k}^{T}-\frac{\lambda_{k} E_{k} E_{k}^{T}}{1+\lambda_{k} x_{k-1}^{T} F_{k-1} x_{k-1}} \\
& \leq \lambda_{k} \delta_{k}^{2}-\frac{\lambda_{k} E_{k} E_{k}^{T}}{1+\lambda_{k} x_{k-1}^{T} F_{k-1} x_{k-1}} \\
& =H_{k}-H_{k-1} \text { (using eqn. 27) }
\end{aligned}
$$

In order to prove equation 25 by induction, assume that

$$
\mathrm{H}_{\mathrm{k}-1}-\tilde{\theta}_{\mathrm{k}-1} \mathrm{~F}_{\mathrm{k}-1}^{-1} \tilde{\theta}_{\mathrm{k}-1}^{\mathrm{T}} \geq 0
$$

Equation 45 can also be written as

$$
\mathrm{H}_{k}-\mathrm{H}_{k-1}-\tilde{\theta}_{k} F_{k}^{-1} \tilde{\theta}_{k}^{T}+\tilde{\theta}_{k-1} F_{k-1}^{-1} \tilde{\theta}_{k-1}^{T} \geq 0
$$

Adding equations 46 and 47 results in 25 . Since by Assumption 3 equation 46 is true for $k=1$, the proof is complete.

2) The two expressions in equation 28 can be readily obtained from equation 14.

3) Introducing equations 15 and 27 into equation 30 and using equation 12 gives

$$
v_{k}-v_{k-1}=\lambda_{k}\left\{\delta_{k}^{2} n-\frac{w_{k}^{2}}{1+\lambda_{k} x_{k-1}^{T} F_{k-1} x_{k-1}}\right\}
$$

In view of the way the dead zone (eqn. 14) was defined, $v_{k}=v_{k-1}$ 
for $w_{k}^{2} \leq \delta_{k}^{2}(1+\alpha) n$. Study the case $w_{k}^{2}>\delta_{k}^{2}(1+\alpha) n$. From equation 48 and using equation 28

$$
\begin{aligned}
& v_{k}-v_{k-1} \leq \lambda_{k}\left\{\delta_{k}^{2} n-w_{k}^{2} /(1+\alpha)\right\} \\
& \leq \frac{\lambda_{k}}{(1+\alpha)}\left\{\delta_{k}^{2}(1+\alpha) n-w_{k}^{2}\right\} \\
& \leq \frac{\alpha\left[\left|w_{k}\right|-\delta_{k}((1+\alpha) n)^{1 / 2}\right]}{(1+\alpha)\left(1+x_{k-1}^{T} F_{k-1} x_{k-1}\right)\left|w_{k}\right|}\left\{\left(\delta_{k}((1+\alpha) n)^{1 / 2}-\left|w_{k}\right|\right)\left(\delta_{k}((1+\alpha) n)^{1 / 2}+\left|w_{k}\right|\right)\right\} \\
& \leq-\frac{\alpha\left[\left|w_{k}\right|-\delta_{k}((1+\alpha) n)^{1 / 2}\right]^{2}}{(1+\alpha)\left(1+x_{k-1}^{T} F_{k-1} x_{k-1}\right)}
\end{aligned}
$$

4) Equation 49 holds for $w_{k}^{2}>\delta_{k}^{2}(1+\alpha) n$, otherwise $v_{k}=v_{k-1}$.

Then $v_{k}$ is a positive nonincreasing sequence and therefore it converges. Equation 31 follows from equation 49.

5) From equation 15 it is noted that $F_{k} \leq F_{k-1}$. Then $z^{T} F_{k} z$, where $z$ is any constant vector, is a positive nonincreasing function and thus converges. To complete the proof it is shown that any element of $F_{k}$ can be expressed as a combination of quadratic terms of the form $z^{T} F_{k} z$. Define $v_{i}$ as the vector of appropiate dimension whose elements are all zero except for the i-th element which is equal to 1 . Then

$F_{k}(i, j)=v_{i}^{T} F_{k} v_{j}=\left[\left(v_{i}+v_{j}\right)^{T} F_{k}\left(v_{i}+v_{j}\right)-v_{i}^{T} F_{k} v_{i}-v_{j}^{T} F_{k} v_{j}\right] / 2$ 
6) Define $\tau$ as those time instants $k$ at which $w_{k}^{2}>\delta_{k}^{2}(1+\alpha) n$. From equation 14

$$
\begin{aligned}
\lambda_{1} x_{1-1}^{T} F_{1-1} x_{1-1} & \leq \frac{\alpha x_{1-1}^{T} F_{i-1} x_{1-1}}{1+x_{1-1}^{T} F_{1-1} x_{1-1}} \\
& \leq \frac{\alpha}{1+\left(x_{1-1}^{T} F_{1-1} x_{1-1}\right)^{-1}} \\
& \leq \frac{\alpha}{1+\left(\operatorname{tr} F_{0}\right)^{-1}}=\frac{\alpha \operatorname{tr} F_{0}}{1+\operatorname{tr} F_{0}}
\end{aligned}
$$

where the fact that $F_{i-1} \leq F_{0}$ (eqn. 15) and $x_{i-1} \leq 1$ (eqn. 10a) was used. Introducing equation 50 into equation 48

$$
\begin{aligned}
& \sum_{k=1}^{\infty} v_{k}-v_{k-1}=v_{\infty}-v_{0} \\
& \quad \leq \sum_{i \in \tau} \lambda_{i}\left\{\delta_{i}^{2} n-\frac{w_{i}^{2}}{1+\frac{\alpha \operatorname{tr} F_{0}}{1+\operatorname{tr} F_{0}}}\right\} \\
& \leq \sum_{i \in \tau} \lambda_{i} \frac{1+\operatorname{tr} F_{0}}{1+\operatorname{tr} F_{0}(1+\alpha)}\left\{\delta_{i}^{2} n\left[1+\frac{\alpha \operatorname{tr} F_{0}}{1+\operatorname{trF} F_{0}}-w_{i}^{2}\right]\right. \\
& \leq \frac{1+\operatorname{tr} F_{0}}{1+\operatorname{tr} F_{0}(1+\alpha)} \sum_{i \in \tau} \lambda_{i}\left\{\delta_{i}^{2} n\left[1+\frac{\alpha \operatorname{tr} F_{0}}{1+\operatorname{tr} F_{0}}\right]-\delta_{i}^{2}(1+\alpha) n\right\}
\end{aligned}
$$


$\leq \frac{1+\operatorname{tr} F_{0}}{1+\operatorname{tr} F_{0}(1+\alpha)} \sum_{i \in \tau} \lambda_{i} \delta_{i}^{2} n \alpha\left(\frac{-1}{1+\operatorname{tr} F_{0}}\right)$

Since $\lambda_{i}=0$ for $i \notin \tau$ and $\delta_{k}$ is bounded, it finally follows

$$
\sum_{k=1}^{\infty} \lambda_{k} \delta_{k}^{2}=\sum_{1 \in \tau} \lambda_{k} \delta_{k}^{2} \leq v_{0}\left(1+\operatorname{trF} F_{0}(1+\alpha)\right) / n \alpha
$$

7) Combining equations 40 and 41 produces

$$
\begin{aligned}
\tilde{\theta}_{k} F_{k}^{-1} & =\tilde{\theta}_{k-1}\left(F_{k-1}^{-1}+\lambda_{k} x_{k-1} x_{k-1}^{T}\right)-\lambda_{k} E_{k} x_{k-1}^{T} \\
& =\tilde{\theta}_{k-1} F_{k-1}^{-1}-\lambda_{k} \underline{N}_{k} x_{k-1}^{T} \quad \text { (using eqn. 43) } \\
& =\tilde{\theta}_{0} F_{0}^{-1}-\sum_{1=1}^{k} \lambda_{1} \underline{N}_{1} x_{1-1}^{T}
\end{aligned}
$$

or equivalently using equation 26

$$
\theta_{k}=\theta-\left[\tilde{\theta}_{0} F_{0}^{-1}-\sum_{1=1}^{k} \lambda_{1} \underline{N}_{1} x_{1-1}^{T}\right] F_{k}
$$

From equation 32, $\sum \lambda_{1} \delta_{1}^{2}$ is a bounded, nonincreasing positive sequence and thus, it converges (ref. 28). Since $\delta \geq \mu>0$ (eqn. 13), $\sum \lambda_{i}$ is also bounded and thus converges too. Since the elements of $\underline{N}_{1} x_{i-1}^{T}$ are all bounded then $\sum \lambda_{1} \underline{N}_{i} x_{1-1}^{T}$ converges. Therefore from the expressions above and since $F_{\mathbf{k}}$ converges, it is concluded that $\theta_{k}$ converges. 
From equation 26

$$
\theta_{k}=\theta+\left(-\tilde{\theta}_{k}\right)
$$

Then using equation B. 1 in Appendix B

$$
\theta_{k} \theta_{k}^{T} \leq 2\left(\theta \theta^{T}+\tilde{\theta}_{k} \tilde{\theta}_{k}^{T}\right)
$$

From equation 25 and some properties of positive definite matrices ( see Appendix B ).

$$
\begin{aligned}
\left(\operatorname{trH}_{k}\right) I & \geq H_{k} \\
& \geq \tilde{\theta}_{k} F_{k}^{-1} \tilde{\theta}_{k}^{T} \\
& \geq \tilde{\theta}_{k}\left[I \lambda_{\min } F_{k}^{-1}\right] \tilde{\theta}_{k}^{T} \\
& \geq \tilde{\theta}_{k} \tilde{\theta}_{k}^{T}\left(\operatorname{trF}_{k}\right)^{-1}
\end{aligned}
$$

On the other hand

$$
\begin{aligned}
\left(\operatorname{trF}_{k}\right)\left(\operatorname{trH} H_{k}\right) & \leq\left(\left[\operatorname{trF}{ }_{k}+\operatorname{trH} H_{k}\right] / 2\right)^{2} \\
& \leq v_{k}^{2} / 4 \text { ( using eqn. 30) } \\
& \leq v_{0}^{2} / 4 \text { (using eqn. 29) }
\end{aligned}
$$

Substituting equation 53 into equation 52 gives

$$
\tilde{\theta}_{k} \tilde{\theta}_{k}^{T} \leq I V_{k}^{2} / 4 \leq I V_{0}^{2} / 4
$$

In order to obtain a bound on $\theta^{\boldsymbol{T}^{T}}$ we recall that asssumption 3 holds for any initial parameter estimate. Therefore equation 54 should also hold for a 0 initial estimate and thus

$$
\theta \theta^{T} \leq \mathrm{I} \mathrm{V}_{0}^{2} / 4
$$


Equation 33 follows after introducing equations 54 and 55 into equation 51 .

8) From equations 33 and 17

$$
\begin{aligned}
\theta_{k} \theta_{k}^{T} & =\left[\begin{array}{llll} 
& B_{k}: & D_{k}
\end{array}\right]\left[\begin{array}{c}
B_{k}^{T} \\
D_{k}^{T}
\end{array}\right] \\
& =B_{k} B_{k}^{T}+D_{k} D_{k}^{T} \leq V_{0}^{2} I
\end{aligned}
$$

Define

$$
\beta_{k}^{*}=\tilde{\theta}_{k}\left(L_{k}^{T}\right)^{-1}
$$

Note that from equations $18,25,29,30$ and the above

$$
\begin{aligned}
\beta_{k}^{*} \beta_{k}^{* T} & =\tilde{\theta}_{k} F_{k}^{-1} \tilde{\theta}_{k}^{T} \\
& \leq H_{k} \leq I \operatorname{tr} H_{k} \\
& \leq I V_{k} \leq I V_{0}
\end{aligned}
$$

Note also that $\beta_{k}^{*}$ in equation 57 is such that

$$
\theta=\theta_{k}+\beta_{k}^{*} L_{k}^{T}
$$

The expression above can be partitioned by using equations $8 a, 17$ and 20 to obtain

$$
B=B_{k}+\beta_{k}^{*}\left(L_{k}^{1}\right)^{T}
$$

Using equation B. 1 


$$
\begin{aligned}
B^{T} B & \leq 2\left[B_{k}^{T} B_{k}+L_{k}^{1} \beta_{k}^{* T} \beta_{k}^{*} L_{k}^{1}{ }^{T}\right] \\
& \leq 2\left[S_{k}^{T} S_{k}+L_{k}^{1} \beta_{k}^{* T} \beta_{k}^{*} L_{k}^{1}{ }^{T}\right] \quad \text { (using eqn 19) } \\
& \leq 2\left[S_{k}^{T} S_{k}+V_{0} L_{k}^{1} L_{1}^{1}\right] \quad \text { (using eqn. 58) }
\end{aligned}
$$

In the last equation the fact that $\beta_{k}^{* T} \beta_{k}^{*}$ and $\beta_{k}^{*} \beta_{k}^{* T}$ have the same nonzero eigenvalues was also used (see Appendix $B$ ). Assume that $S_{k}=\underline{S}_{k} \underline{S}_{k}^{T}$ then

$$
\begin{aligned}
S_{k}^{T} S_{k} & =\underline{S}_{k}\left(\underline{S}_{k}^{T} \underline{S}_{k}\right) \underline{S}_{k}^{T} \\
& \leq\left[\lambda_{\max } S_{k}\right] S_{k} \leq\left[\lambda_{\max } S_{k}^{T} S_{k}\right]^{1 / 2} S_{k} \\
& \leq\left[\lambda_{\max } B_{k}^{T} B_{k}\right]^{1 / 2} S_{k} \quad \text { (using eqn. 19) } \\
& \leq v_{0} S_{k} \quad \text { (using eqn. 56) }
\end{aligned}
$$

Introducing equation 62 into equation 61 and in view of Assumption 2

$$
b_{0} I \leq B^{T} B \leq 2 V_{0}\left[S_{k}+L_{k}^{1} L_{k}^{1^{T}}\right]
$$

From equations $17,20,22$ and 23

$$
\underline{B}_{k}=B_{k}+\beta_{k} L_{k}^{1}
$$

Introducing equations 19 and 21 into the above 


$$
\underline{B}_{k}=Q_{k}\left[S_{k}+L_{k}^{1} L_{k}^{1^{T}}\right]
$$

Thus

$$
\begin{aligned}
\underline{B}_{k}^{T} B_{k} & =\left[S_{k}+L_{k}^{1} L_{k}^{1^{T}}\right]^{T}\left[S_{k}+L_{k}^{1} L_{k}^{1^{T}}\right] \quad \text { (using eqn. 19) } \\
& \geq I b_{0}^{2} / 4 V_{0}^{2} \quad \text { (using eqn. 63) }
\end{aligned}
$$

9)

$$
\begin{aligned}
\left(\underline{B}_{k}^{-1} \underline{D}_{k}\right)^{T}\left(\underline{B}_{k}^{-1} \underline{D}_{k}\right) & =\underline{D}_{k}^{T}\left(\underline{B}_{k} \underline{B}_{k}^{T}\right)^{-1} \underline{D}_{k} \\
& \leq \underline{D}_{k}^{T} \underline{D}_{k} \lambda_{\max }\left(\underline{B}_{k} \underline{B}_{k}^{T}\right)^{-1} \\
& \leq \underline{D}_{k}^{T} \underline{D}_{k}\left[\lambda_{\min } \underline{B}_{k} \underline{B}_{k}^{T}\right]^{-1} \\
& \leq \underline{D}_{k}^{T} \underline{D}_{k} 4 v_{0}^{2} / b_{0}^{2} \quad \text { (using eqn. 64) }
\end{aligned}
$$

From equations 17, 20, 22 and 23

$$
\underline{D}_{k}=D_{k}+\beta_{k} L_{k}^{2^{T}}
$$

Then

$$
\begin{aligned}
& \underline{D}_{k}^{T} \underline{D}_{k} \leq 2\left[D_{k}^{T} D_{k}+L_{k}^{2} \beta_{k}^{T} \beta_{k} L_{k}^{2^{T}}\right] \text { (using eqn. B. 1) } \\
& \leq 2\left[v_{0}^{2} I+L_{k}^{2} L_{k}^{1{ }^{T}} L_{k}^{1} L_{k}^{2^{T}}\right] \text { (using eqns.21 and 56) } \\
& \leq 2\left[v_{0}^{2}+\left(\lambda_{\max } L_{k}^{1^{T}} L_{k}^{1}\right)\left(\lambda_{\max } L_{k}^{2} L_{k}^{2^{T}}\right)\right] I \\
& \leq 2\left[\mathrm{v}_{0}^{2}+\left(\lambda_{\max } \mathrm{L}_{\mathrm{k}}^{\mathrm{T}} \mathrm{L}_{\mathrm{k}}^{1}+\lambda_{\max } \mathrm{L}_{\mathrm{k}}^{\mathrm{L}^{\mathrm{T}}} \mathrm{L}_{\mathrm{k}}^{2}\right)^{2} / 4\right] \mathrm{I} \\
& \leq 2\left[v_{0}^{2}+\left(2 \lambda_{\max } L_{k}^{T} L_{k}\right)^{2} / 4\right] \text { I (see below) }
\end{aligned}
$$




$$
\leq 4 \mathrm{v}_{0}^{2} \mathrm{I} \text { (using eqns. 18, } 29 \text { and 30) }
$$

where the fact that ( see eqn. 20)

$$
L_{k}^{T} L_{k}=L_{k}^{1^{T}} L_{k}^{1}+L_{k}^{2^{T}} L_{k}^{2}
$$

was used. The result follows from equations 65 and 66

10) Define the modified prediction error

$$
\begin{aligned}
\underline{E}_{k} & =\underline{Y}_{k}-\Theta_{k-1} x_{k-1} \\
& =E_{k}-\beta_{k-1} L_{k-1}^{T} x_{k-1} \text { (using eqns. } 11 \text { and 22) }
\end{aligned}
$$

Therefore, using equation B. 1

$$
\underline{E}_{k}^{T} \underline{E}_{k} \leq 2\left(\underline{E}_{k}^{T} E_{k} \pm \underline{x}_{k-1}^{T} \underline{L}_{k-1} \beta_{k-1}^{T} \beta_{k-1} \underline{L}_{k-1}^{T} \underline{x}_{k-1} 2\right.
$$

But from equations 19 and 21

$$
\begin{aligned}
L_{k-1} \beta_{k-1}^{T} \beta_{k-1} L_{k-1}^{T} & =L_{k-1} L_{k-1}^{1^{T}} L_{k-1}^{1} L_{k-1}^{T} \\
& \leq L_{k-1} L_{k-1}^{T} L_{k-1} L_{k-1}^{T} \text { (using eqn. 67) } \\
& \leq F_{k-1}^{2} \text { (using eqn. 18) }
\end{aligned}
$$

Introducing equation 71 into equation 70 and taking into account equation 12 we get

$$
E_{k}^{T} E_{k} \leq 2 w_{k}^{2}
$$

Using equation $8 b, 10$ and 23 , equation 68 can also be rewritten as 


$$
\underline{E}_{k}=\frac{1}{1+\left\|\phi_{k-1}\right\|}\left\{y_{k}-\left[\underline{B}_{k-1}, \underline{D}_{k-1}\right]\left[\begin{array}{c}
U_{k-1} \\
y_{k-1}
\end{array}\right]\right\}
$$

Using the control law in equation 24, equation 73 reduces to

$$
E_{k}=\frac{Y_{k}}{1+\left\|\phi_{k-1}\right\|}
$$

Combining equations 72 and 74

$$
\begin{aligned}
2\left[w_{k}^{2}-\delta_{k}^{2}(1+\alpha) n\right] & \geq E_{k}^{T} E_{k}-2 \delta_{k}^{2}(1+\alpha) n \\
& \geq \frac{Y_{k}^{T} Y_{k}}{\left(1+\left\|\phi_{k-1}\right\|\right)^{2}}-2 \delta_{k}^{2}(1+\alpha) n
\end{aligned}
$$

From equations $8 \mathrm{~b}$ and 24 it follows

$$
\begin{aligned}
\left\|\phi_{k}\right\|^{2} & =\left\|Y_{k}\right\|^{2}+\left\|U_{k}\right\|^{2} \\
& \leq\left\|Y_{k}\right\|^{2}+Y_{k}^{T}\left(\underline{B}_{k}^{-1} \underline{D}_{k}\right)^{T} \underline{B}_{k}^{-1} \underline{D}_{k} Y_{k} \\
& \leq(1+m)\left\|Y_{k}\right\|^{2} \quad \text { (see eqn. 34a) }
\end{aligned}
$$

On the other hand, using equations 8 and 9 it is seen that

$$
\left\|Y_{k}\right\| \leq(\|\theta\|+\mu)\left\|\phi_{k-1}\right\|+\eta
$$

Assume that $\left\|Y_{k}\right\|$ grows unbounded. Define a subsequence $t_{n}$ along which $\left\|Y_{t_{n}}\right\| \rightarrow \infty$ and 


$$
\left\|Y_{t_{n}}\right\| \geq \max _{1<t_{n}}\left\|Y_{i}\right\|
$$

Then using equation 76 and the above gives

$$
\begin{aligned}
\frac{\left\|Y_{t_{n}}\right\|}{1+\left\|\phi_{t_{n}-1}\right\|} & \geq\left\|Y_{t_{n}-1}\right\| /\left[1+(m+1)^{1 / 2}\left\|Y_{t_{n}-1}\right\|\right] \\
& \geq 1 /\left[(m+1)^{1 / 2}+\left\|Y_{t_{n}-1}\right\|^{-1}\right]
\end{aligned}
$$

Introducing equations 79 and 13 into equation 75

$$
\begin{aligned}
2\left[w_{t}^{2}-\delta_{t}^{2}(1+\alpha) n\right] \geq & {\left[(m+1)^{1 / 2}+\left\|Y_{t_{n}-1}\right\|^{-1}\right]^{-2}-} \\
& -2(1+\alpha) n\left[\mu+\eta /\left(1+\left\|\phi_{t_{n}-1}\right\|\right)\right]^{2}
\end{aligned}
$$

$\left\|Y_{t_{n}}\right\| \rightarrow$ implies $\left\|\phi_{t_{n}-1}\right\| \rightarrow \infty$ and $\left\|Y_{t_{n}-1}\right\| \rightarrow \infty$ as can be seen from equations 76 and 77 . Therefore the RHS of equation 80 converges to (see also eqn. 35)

$$
(1+m)^{-1}-2(1+\alpha) n \mu^{2}=\frac{1-(1-\varepsilon)^{2}}{m+1}>0
$$

Since this limit is strictly positive it contradicts the fact that the LHS of equation 80 satisfies equation 31 . Therefore $\left\|Y_{k}\right\|$ should remain bounded and so should $\left\|\phi_{k}\right\|$ and $\left\|U_{k}\right\|$ (see eqn. 76). In order to obtain an explicit bound for $\left\|Y_{k}\right\|$ consider equation 75 again to obtain 
$2\left[w_{k}^{2}-\delta_{k}^{2}(1+\alpha) n\right] \geq z_{k}\left[-(2 n(1+\alpha))^{1 / 2} \delta_{k}+\left\|Y_{k}\right\| /\left(1+\left\|\phi_{k-1}\right\|\right)\right]$

where

$$
z_{k}=\left[(2 n(1+\alpha))^{1 / 2} \delta_{k}+\left\|Y_{k}\right\| /\left(1+\left\|\phi_{k-1}\right\|\right)\right]
$$

Introducing equation 13 into equation 81 gives

$2\left[w_{k}^{2}-\delta_{k}^{2}(1+\alpha) n\right] \geq$

$$
\begin{aligned}
& {\left[\left\|Y_{k}\right\|-(2 n(1+\alpha))^{1 / 2}\left\{\eta+\mu\left(1+\left\|\phi_{k-1}\right\|\right)\right\}\right] z_{k} /\left(1+\left\|\phi_{k-1}\right\|\right)} \\
& \geq\left[\left\|Y_{k}\right\|-(2 n(1+\alpha))^{1 / 2}\left\{\eta+\mu+\mu(1+m)^{1 / 2}\left\|Y_{k-1}\right\|\right\}\right] z_{k} /\left(1+\left\|\phi_{k-1}\right\|\right) \\
& \text { (using eqn. 76) }
\end{aligned}
$$

$\geq\left[\left\|Y_{k}\right\|-(1-\varepsilon)\left\|Y_{k-1}\right\|-(2 n(1+\alpha))^{1 / 2}(\eta+\mu)\right] z_{k} /\left(1+\left\|\phi_{k-1}\right\|\right)$

(using eqn. 35)

From equations 13, 82 and the boundedness of $\left\|\phi_{k-1}\right\|$ it is clear that $z_{k} /\left(1+\left\|\phi_{k-1}\right\|\right)$ is strictly positive. Therefore, from equation 31 and the above we conclude that

$\lim _{k \rightarrow \infty} \sup \left[\left\|Y_{k}\right\|-(1-\varepsilon)\left\|Y_{k-1}\right\|-(2 n(1+\alpha))^{1 / 2}(\eta+\mu)\right] \leq 0$

The result follows.

\section{Concluding Remarks}

This report presented an adaptive scheme that achieves regulation without resorting to any persistent excitation condition. The proposed algorithm can be used to control minimum phase or nonminimum phase plants subject to bounded disturbances and unmodeled dynamics.

An explicit modification for the parameter estimates was presented that avoids having any ill-conditionned matrix in the control law. This allowed the giving of explicit bounds for the 
plant output.

The proposed technique can also be used in adaptive control of multivariable systems whenever the estimate of a matrix coefficient is required to be non-singular.

\section{Appendix A}

Proof of Lemma 1

Iterating equation $1 \mathrm{a}$

$$
\begin{aligned}
x(t+i)=A^{1} x(t) & +A^{i-1} b u(t)+\ldots+ \\
& +A^{i-1} b^{\prime} v^{\prime}(t)+\ldots u(t+i-1)+
\end{aligned}
$$

for $1=n$

$$
x(t+n)=A^{n} x(t)+\mathscr{E U}(t)+\mathscr{C}^{\prime} V^{\prime}(t)
$$

with $6, C^{\prime}, U(t)$ and $V^{\prime}(t)$ as defined in Lemma 1. Premultiplying equation $\mathrm{A} .1$ by $c^{\mathrm{T}}$ and using equation $1 \mathrm{~b}$ gives

or

$$
Y(t+n)=O x(t)+G U(t)+G^{\prime} V^{\prime}(t)+V^{\prime \prime}(t)
$$

$$
x(t)=O^{-1}\left[Y(t+n)-G U(t)-G^{\prime} V^{\prime}(t)-V^{\prime \prime}(t)\right]
$$

with G, G', $Y$ and $V^{\prime \prime}$ as defined in Lemma 1. Iterating equation A. 2 produces

$$
x(t+n)=A^{2 n} x(t-n)+A^{n} C U(t-n)+A^{n} C^{\prime} V^{\prime}(t-n)+C U(t)+C^{\prime} V^{\prime}(t) \quad(A .4)
$$

Substitution of equation A.4 in equation A. 3 finally leads to equation 2 with all the variables as defined in Lemma 1 


\section{Appendix B}

This Appendix is intended to recall some results from matrix theory that are used in the report. Refer to References 29, 30 and 31 for a more detailed analysis.

1.- For any matrices $A$ and $B$ it follows that

$$
\begin{aligned}
(A+B)(A+B)^{T} & \leq(A+B)(A+B)^{T}+(A-B)(A-B)^{T} \\
& \leq 2\left(A A^{T}+B B^{T}\right)
\end{aligned}
$$

2.- For any positive semi-definite matrix $F \geq 0$ it is noted that

1) $\left(\lambda_{\min } F\right) I \leq F \leq\left(\lambda_{\max } F\right) I \leq(t r F)$ I

ii) $\exists L$ such that $F=L L^{T}$

iii) $\lambda_{\max } F^{-1}=\left[\lambda_{\min } F\right]^{-1}$

3. - For any matrix $A, A^{T} A$ and $A A^{T}$ have no negative eigenvalues and have the same nonzero eigenvalues. (ref. 30 page 182)

Sketch of the proof

Let $\lambda_{1}$ and $v_{1}$ be the eigenvalue and corresponding eigenvector of $\mathrm{AA}^{\mathrm{T}}$, then

$$
\mathrm{AA}^{\mathrm{T}} \mathrm{v}_{\mathbf{i}}=\lambda_{1} \mathrm{v}_{\mathbf{i}}
$$

premultiplying by $A^{T}$

$$
A^{T} A\left(A^{T} v_{i}\right)=\lambda_{1}\left(A^{T} v_{i}\right)
$$

Premultiplying equation $B .2$ by $v_{i}^{T}$ results in $\left\|A^{T} v_{i}\right\|^{2}=\lambda_{i}\left\|v_{i}\right\|^{2}$. Then $A^{T} v_{1} \neq 0$ for $\lambda_{i} \neq 0$ and therefore $A^{T} v_{1}$ is the eigenvector corresponding to the eigenvalue $\lambda_{1}$ of $A^{T} A$. 
4. - Any matrix $A$ can be expressed as $A=Q S$ where $Q^{T} Q=I$ and $\mathrm{S} \geq 0$ (ref. 29 p286)

Sketch of the proof

Let $v_{i}$ and $\lambda_{i}$ such that

$$
A^{T} A v_{i}=\lambda_{i}^{2} v_{i} \quad v_{i}^{T} v_{j}=\delta_{i j}
$$

Then $\exists z_{1}, z_{1}^{T} z_{j}=\delta_{i j}$ such that

$$
A v_{1}=\lambda_{i} z_{i}
$$

Define

$$
\begin{aligned}
& Q=\sum z_{j} v_{j}^{T} \quad \Rightarrow \quad Q v_{i}=z_{i} \\
& S=\sum \lambda_{j} v_{j} v_{j}^{T} \quad \Rightarrow \quad S v_{i}=\lambda_{i} v_{i}
\end{aligned}
$$

Then from equations B. 4 through B. 6

$$
A v_{1}=\lambda_{1} Q v_{1}=Q S v_{i} \Rightarrow A=Q S
$$

Furthermore, from equation B.6) $S \geq 0$ and from equation B. 5

$$
Q^{T} Q=\sum_{i} v_{i} z_{i}^{T} \sum_{j} z_{j} v_{j}^{T}=\sum_{i} v_{i} v_{i}=I
$$

\section{References}

1 Goodwin G.C.; Ramadge P.J.; and Caines P.E. : Discrete-Time Multivariable Adaptive Control. IEEE TAC vol. AC-24, pp 449-456, June 1980.

2 Morse A.S.: Global Stability of Parameter Adaptive Control Systems. IEEE TAC vol. 25, pp 433-439, June 1980.

3 Narendra K.S.; and Lin Y. H.: Stable Discrete Adaptive Control" IEEE TAC vol. 25, pp456-461, June 1980. 
4 Landau I.D.; Lozano-Leal R.: Unification of Discrete-Time Explicit Model Reference Adaptive Control Designs. Automatica vol. 17, pp 595, 1981.

5 Narendra K.S.; Lin Y.H. ; and Valavani L.S.: Stable Adaptive Control Design, Part II : Proof of stability. IEEE TAC vol. 25, pp 440-449, 1980.

6 Ortega R.; Praly L.; and Landau I.D.: Robustness of Discrete-Time Direct Adaptive Ccontrollers. IEEE TAC vol. 30, pp 1179-1187, Dec. 1985.

7 Ioannou P.A, ; and Tsakalis K.S. : A Robust Direct Adaptive Controller. IEEE TAC vol. 31, pp 1033-1043, Nov. 1986.

8 Kreisselmeier G.; and Anderson B.D.O.: Robust Model Reference Adaptive Control. IEEE TAC vol. 31, pp 127-133, Feb. 1986.

9 Goodwin G.C.; Lozano-Leal R.; Mayne D.Q.; and Middleton R. H.: Rapprochement Between Continuous and Discrete Model Reference Adaptive Control. Automatica vol. 22, pp. 199-207, 1986.

10 Sastry S.S.: Model Reference Adaptive Control Stability, Parameter Convergence and Robustness. IMAJ Contr. and Inform. vol. 1 pp 27-66, 1984.

11 Astrom K. J.; Hagander P.; and Sternby J.: Zeros of Sampled Systems. Automatica vol 22, pp 199-207, 1986.

12 Anderson B.D.O.; and Johnstone R.M.: Global Pole Positioning. IEEE TAC vol. 30, pp 11-21, Jan. 1985.

13 Elliott H; Cristi R.; and Das M.: Global Stability of Adaptive Pole Placement Algorithms. IEEE TAC vol. 30, pp 348-356, April 1985.

14 Goodwin G.C.; and Teoh E.K.: Persistency of Excitation in Presence of Possibly Unbounded Signals:. IEEE TAC vol 30, pp 595-597, June 1985.

15 Kreisselmeier G.; and Smith M.C.: Stable Adaptive Regulation of Arbitrary n-th Order Plants. IEEE TAC vol. 31, pp 299-305, April 1986.

16 de Larminat P.: On the Stabilization Condition in Indirect Adaptive Control:. Automatica vol. 20, pp793-795, 1984.

17 Lozano-Leal R.; and Goodwin G.C.: A Globally Convergent Adaptive Pole Placement Algorithm Without a Persistency of Excitation Requirement. IEEE TAC vol 30, pp 795-797, Aug. 1985. 
18 Goodwin G.C.; and Sin K.S.: . Adaptive Filtering Prediction and Control. Englewood Cliffs, NJ Prentice Hall, 1984.

19 Goodwin G.C.; Hill D.; Mayne D.Q.; and Middleton R.H.: Adaptive Robust Control (Convergence, Stability and Performance). Proceedings of the 25th IEEE CDC, pp 462-467, Athens 1986.

20 Narendra K.S. ; and Annaswamy A.M.: A New Adaptive Law for Robust Adaptation Without Persistent Excitation. IEEE TAC vol. 32, pp 134-145, Feb. 1987.

21 Kreisselmeier G.: Adaptive Control via Adaptive Observation and Asymptotic Feedback Matrix Synthesis. IEEE TAC vol. 25 pp 717-722, Aug. 1980.

22 Goodwin G.C. ; and Sin K.S.: Adaptive Control of Nonminimum Phase Systems. IEEE TAC vol. 26, pp 478-483, April 1981.

23 de Larminat P.; Raynaud H.F.: A Robust Solution to the Stabilisability Problem in Indirect Passive Adaptive Control. Proceedings of the 25th IEEE CDC, pp 468-473, Athens 1986.

24 M'Saad M. ; Ortega R. ; and Landau I.D.: Adaptive Controllers for Discrete-Time Systems with Arbitrary Zeros - An Overview. Automatica vol. 21, pp 413-415, 1985.

25 Bai E.W.; and Sastry S.S.: Discrete Time Adaptive Control Utilizing Prior Information. IEEE vol 31, pp 779-782, Aug. 1986.

26 Feuer A.: A Parameterization for Model Reference Adaptive Pole-Placement. IEEE TAC vol 31, pp 782-785, Aug. 1986.

27 Praly L.: Towards a Globally Stable Direct Adaptive Control Scheme for not Necessarily Minimum Phase Systems. IEEE Trans. on Automatic Control Vol AC-29, No 10, Oct 1984.

28 Knopp K.:. Infinite Sequences and Series. New York Dover Publications, 1956.

29 Gantmacher F.R.: Theory of Matrices. Chelsea Publishing Co., New York, 1959, Vol I.

30 Lancaster P.; Tismenetsky M.: The Theory of Matrices. Academic Press 1985.

31 Shieh L.S.; Lian S.R.; and Mcinnis B.: Fast and Stable Algorithms for Computing the Principal Square Root of a Complex Matrix. IEEE Trans. on Automatic Control Vol. AC-32, No 9, pp 820-822, Sept. 1987. 
32 Lozano L.R.; and Ortega R.: Reformulation of the Parameter Identification Problem for Systems with Bounded Disturbances. Automatica Vol 23, No 2, pp 247-251, March 1987. 


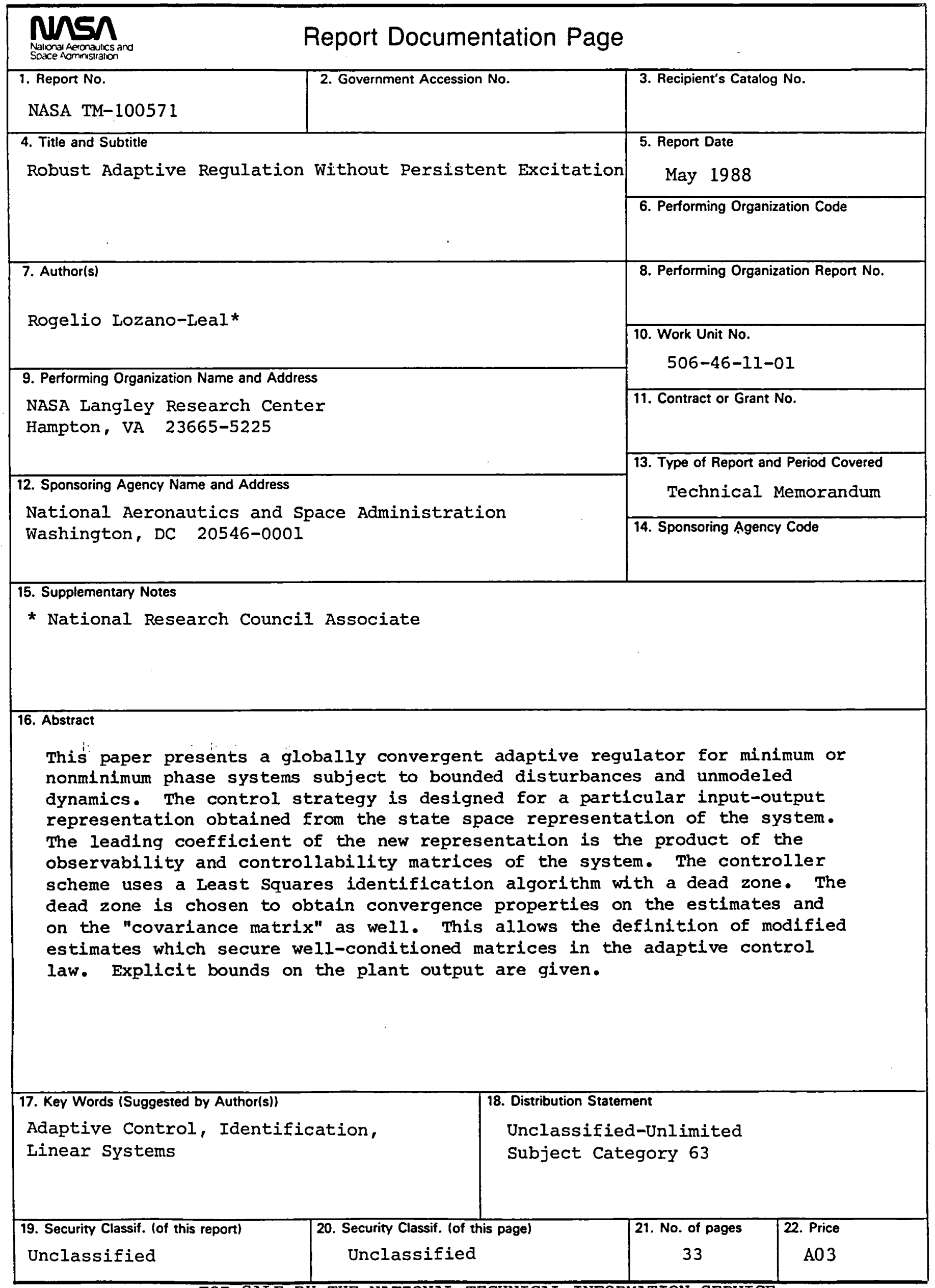

